\title{
Economic evaluation of strategies for managing crying and sleeping problems
}

\author{
S Morris, I St James-Roberts, J Sleep, P Gillham
}

\begin{abstract}
Aims-To estimate the financial cost to the NHS of infant crying and sleeping problems in the first 12 weeks of age and to assess the cost effectiveness of behavioural and educational interventions aimed at reducing infant crying and sleeping problems relative to usual services.

Methods-A cost burden analysis and cost effectiveness analysis were conducted using data from the Crying Or Sleeping Infants (COSI) Study, a three armed prospective randomised controlled trial that randomly allocated 610 mothers to a behavioural intervention $(n=205)$, an educational intervention $(n=202)$, or existing services (control, $n=203$ ). Main outcome measures were annual total cost to the NHS of infant crying and sleeping problems in the first 12 weeks, and incremental cost per interruption free night gained for behavioural and educational interventions relative to control. Results-The annual total cost to the NHS of infant crying and sleeping problems in the first 12 weeks was $£ 65$ million (US $\$ 104$ million). Incremental costs per interruption free night gained for the behavioural intervention relative to control were $£ 0.56$ (US\$0.92). For the educational intervention relative to control they were $£ 4.13$ (US\$6.80).

Conclusions-The annual total cost to the NHS of infant crying and sleeping problems is substantial. In the cost effectiveness analysis, the behavioural intervention incurred a small additional cost and produced a small significant benefit at 11 and 12 weeks of age. The educational intervention incurred a small additional cost without producing a significant benefit.

(Arch Dis Child 2001;84:15-19)
\end{abstract}

Keywords: crying and sleeping problems; cost burden; behavioural programmes; cost effectiveness

Infant crying and sleeping problems are two of the most common issues on which parents seek advice from health care professionals. ${ }^{12} \mathrm{Up}$ to $21 \%$ of UK families report having sought advice for persistent infant crying, ${ }^{23}$ while night waking is an equally common problem as infants get older. ${ }^{4}$ Clearly these problems impose a substantial cost on the UK National Health Service (NHS). However, at the present time no data exist on cost and resource implications.

Evidence suggests that behavioural programmes or improved education for mothers about their babies may reduce infant crying and sleeping problems. Studies conducted previously in this area, while reporting promising findings, have a number of limitations including recruitment of small, selective study populations and short duration of follow up. ${ }^{56}$ Additionally, no evidence is available on the cost effectiveness of these interventions.

\section{Methods}

Our objectives were, firstly, to estimate the cost of infant crying and sleeping problems in the first 12 weeks of age, and secondly, to assess the cost effectiveness of behavioural and educational interventions aimed at reducing infant crying and sleeping problems relative to usual services. The perspective taken was that of the NHS (that is, only costs to the NHS were considered).

\section{THE COSI STUDY}

The analysis was conducted using data from the Crying Or Sleeping Infants (COSI) Study. This has been reported in greater detail elsewhere. ${ }^{7}$ The hypothesis tested was that behaviour intervention or educational support can be used preventively to reduce crying and improve sleeping in 1-12 week old infants compared with existing services. The study design was a three armed prospective controlled trial, in which 610 mothers were randomly allocated to either a behavioural intervention $(\mathrm{n}=205)$, an educational intervention $(\mathrm{n}=202)$, or existing services (control, $\mathrm{n}=203$ ). The trial was conducted in West Berkshire between March 1997 and February 1998.

The behavioural intervention was a modification of the Pinilla and Birch programme to accentuate day/night differences. ${ }^{5}$ It consisted of three components. Firstly, mothers were asked to provide a focal feed for their babies between $10 \mathrm{pm}$ and 12 midnight. Secondly, they were asked not to rock, hold, or feed their babies to sleep and to minimise interaction during the night. Thirdly, once their babies were 3 weeks of age and gaining weight satisfactorily, mothers were asked to lengthen intervals between night feeds by resettling their baby without feeding, thus disassociating night waking from being fed.

The educational intervention comprised an information booklet produced in conjunction with local health visitors. This incorporated current best practice relating to management of crying and sleeping problems. Mothers receiving the educational intervention were also provided with contact details for a dedicated telephone helpline organised by the voluntary support group, CRYSIS.

In the control group, mothers and babies received standard existing services offered by 


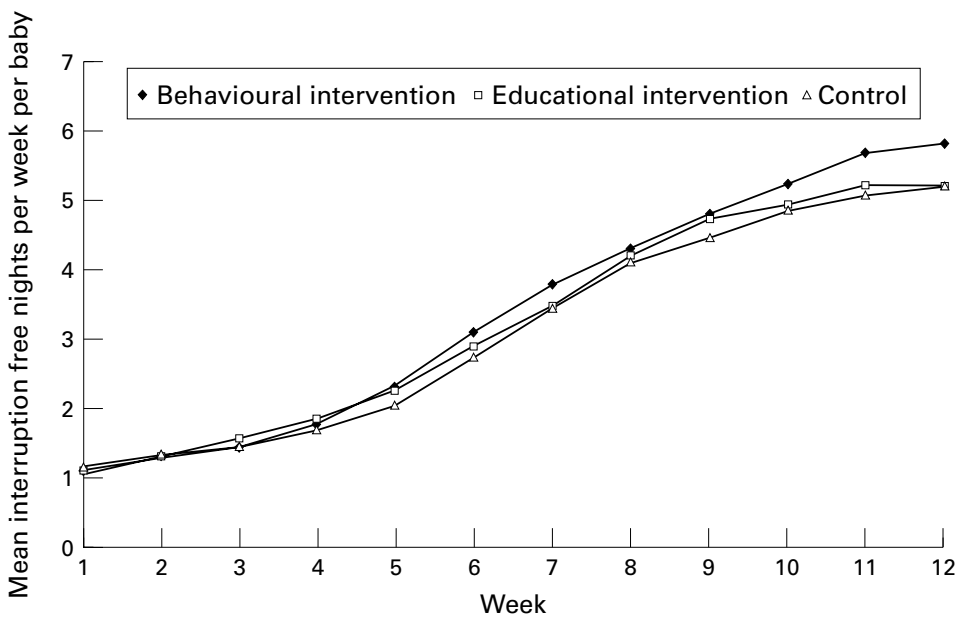

Figure 1 Mean interruption free nights per week per baby during weeks 1-12 for the behavioural intervention $(n=150)$, educational intervention $(n=159)$, and control groups $(n=132)$.

midwives, health visitors, and general practitioners (GPs). These services were available to all trial participants.

MEASURING EFFECTIVENESS

Effectiveness in the cost effectiveness analysis was measured in terms of interruption free nights gained from the behavioural and educational interventions relative to the control. An interruption free night was defined as a night in which parents reported their baby to remain asleep continuously for five hours or more between $10 \mathrm{pm}$ and $6 \mathrm{am}$. This choice of outcome measure reflects infrared video recorded evidence that most infants wake in the night, but return to sleep without disturbing their parents. ${ }^{89}$ The focus in this study was on instances where night waking is detected by parents, usually because of crying, as it is parents who seek NHS help for such problems. Mothers were asked to keep prospective logs of the number of interruption free nights every week across the 12 week period. At weeks 1, 3, 6,9 , and 12 mothers were also asked to record for three consecutive days their baby's night sleeping habits in a diary, independently of the logs described above. The diary data were consistent with the log data, ${ }^{10}$ supporting the use of the prospective logs as a reliable source of effectiveness data in this study.

\section{MEASURING COSTS}

Cost components included were the cost of each intervention and the cost of all contacts with health care professionals where infant crying and sleeping problems were discussed.

The cost per hour of contacts with health care professionals were taken from Netten et $a l .{ }^{11}$ These are national estimates of staff costs in the NHS and include: the cost of wages and salaries; the cost of employers' national insurance and superannuation contributions; preand post-registration initial training costs; ongoing training and qualification costs; clerical and administrative costs (including paperwork); the cost of direct supervision and uniforms; the cost of indirect support services (human resources, finances, and estates); capital overhead costs; and travel costs.

The intervention costs consisted of the mean time spent by health visitors explaining the interventions and the cost of providing the relevant information pack to mothers. As recorded in the trial the mean time spent explaining each intervention to mothers was eight minutes for the behavioural intervention and three minutes for the educational intervention. It was assumed these explanations would in practice be provided by a $G$ grade health visitor at a cost of $£ 63.00$ (US\$98) per hour with a patient, or $£ 1.05$ (US\$1.73) per minute. ${ }^{11}$ On this basis the cost of the health visitor's time spent explaining the interventions was $£ 8.40$ (US\$13.85) for the behavioural intervention and $£ 3.15$ (US\$5.20) for the educational intervention. The cost per information pack was $£ 0.16$ (US $\$ 0.28$ ) for the behavioural intervention (manufacturer's price $£ 800$ (US\$1320) for 5000 packs) and £0.26 (US\$0.43) for the educational intervention ( $£ 1300$ (US\$2145) for 5000). Therefore the average cost of the behavioural intervention was $£ 8.56$ (US $\$ 14.27$ ), and the average cost of the educational intervention was £3.41 (US\$5.62).

Costs arising from contacts with the NHS pertaining wholly or in part to infant crying and sleeping problems included the cost of: mothers' visits to the health visitor at the clinic or surgery; health visitors' or midwives' visits to the mother at home; mothers' telephone calls to the health visitor or midwife; mothers' visits to the GP at the surgery; and GPs' visits to the mothers at home. Mothers were asked to keep prospective logs of the number of contacts that wholly or partly concerned their baby's crying and sleeping problems every week across the 12 week period.

The duration and unit cost of NHS contacts were determined by three surveys conducted before the trial. Firstly, in December 1996 three visits were made to clinics run by two health visitors in Reading and Wokingham. In consultations where mothers asked for advice on their baby's crying and sleeping problems the mean time spent discussing these issues was seven minutes. The cost of a health visitor's time in clinic was $£ 53.00$ (US\$87.50) per hour or $£ 0.88$ (US $\$ 1.45$ ) per minute, ${ }^{11}$ implying a cost per clinic consultation of $£ 6.18$ (US $\$ 10.20$ ).

Secondly, in January 1997, four health visitors working in Reading were asked to record for four weeks the number of telephone contacts with mothers spent discussing their baby's crying and sleeping problems and length of time of these discussions. The mean time was 12 minutes. The cost of a health visitor's time with a patient was $£ 63.00$ (US $\$ 98$ ) per hour or $£ 1.05$ (US $\$ 1.73$ ) per minute, ${ }^{11}$ implying a cost per telephone contact of $£ 12.60$ (US\$20.75).

Thirdly, the same four health visitors were asked to record during the same period the time spent in home visits to mothers discussing infant crying and sleeping problems. For consultations where mothers asked for advice 
Table 1 Mean NHS and non-NHS contacts per baby for the behavioural intervention, educational intervention, and control groups pertaining wholly or in part to infant crying and sleeping problems

\begin{tabular}{|c|c|c|c|c|c|c|c|}
\hline & \multicolumn{3}{|c|}{ Mean (SD) contacts per baby } & \multirow[b]{2}{*}{$\begin{array}{l}\text { Unit cost } \\
(£)\end{array}$} & \multicolumn{3}{|c|}{ Mean $(S D)$ cost per baby $(£)$} \\
\hline & $\begin{array}{l}\text { Behavioural } \\
\text { intervention } \\
(n=159)\end{array}$ & $\begin{array}{l}\text { Educational } \\
\text { intervention } \\
(n=159)\end{array}$ & $\begin{array}{l}\text { Control } \\
(n=148)\end{array}$ & & $\begin{array}{l}\text { Behavioural } \\
\text { intervention } \\
(n=159)\end{array}$ & $\begin{array}{l}\text { Educational } \\
\text { intervention } \\
(n=159)\end{array}$ & $\begin{array}{l}\text { Control } \\
(n=148)\end{array}$ \\
\hline \multicolumn{8}{|l|}{ NHS contacts } \\
\hline Mother visits health visitor at clinic or surgery & $1.35(2.29)$ & $1.59(2.54)$ & $1.12(2.04)$ & 6.18 & $8.37(14.15)$ & $9.81(15.72)$ & $6.93(12.59)$ \\
\hline Health visitor or midwife visits mother at home & $1.81(2.63)$ & $2.11(3.16)$ & $2.07(3.08)$ & 28.23 & $51.02(74.19)$ & $59.44(89.14)$ & $58.54(86.95)$ \\
\hline Mother telephones health visitor or midwife & $0.35(1.02)$ & $0.38(0.94)$ & $0.36(0.75)$ & 12.60 & $4.41(12.81)$ & $4.77(11.78)$ & $4.48(9.50)$ \\
\hline Mother visits GP at surgery & $0.61(1.12)$ & $0.71(1.19)$ & $0.51(0.92)$ & 18.00 & $11.07(21.59)$ & $12.83(21.50)$ & $9.24(16.46)$ \\
\hline GP visits mother at home & $0.16(0.40)$ & $0.14(0.48)$ & $0.19(0.53)$ & 56.00 & $8.75(22.25)$ & $7.70(27.01)$ & $10.90(29.60)$ \\
\hline Total cost & & & & & $83.89(116.92)$ & $95.55(127.88)$ & $90.64(123.30)$ \\
\hline \multicolumn{8}{|l|}{ Non-NHS contacts } \\
\hline Mother contacts friends and relatives & $1.96(4.03)$ & $2.71(6.47)$ & $2.20(4.40)$ & & & & \\
\hline Mother telephones CRYSIS helpline & $0.01(0.11)$ & $0.02(0.18)$ & $0.02(0.14)$ & & & & \\
\hline Mother telephones other helplines & $0.17(0.50)$ & $0.16(0.61)$ & $0.09(0.43)$ & & & & \\
\hline
\end{tabular}

Mean costs per baby may not sum exactly due to rounding error.

on these problems the mean time was 22 minutes. The cost of a health visitor's home visit was $£ 77.00$ (US\$127.05) per hour or $£ 1.28$ (US\$2.11) per minute, ${ }^{11}$ implying a cost per home visit of $£ 28.23$ (US $\$ 46.62$ ).

The cost of a mother's visit to the GP at the surgery pertaining to infant crying and sleeping problems was $£ 18.00$ (US\$29.70). ${ }^{11}$ The cost of a GP's visit to the mothers at home was $£ 56.00$ (US $\$ 92.40) .{ }^{11}$ It was assumed for both these contacts that the entire contact would be devoted to infant crying and sleeping.

Data were also collected on the number of non-NHS contacts pertaining wholly or in part to infant crying and sleeping problems. These comprised contacts with friends and relatives and telephone calls to CRYSIS and other helplines.

Data were also collected on the use of medications prompted by the baby's crying and/or unsettled behaviour. Mothers were asked to keep prospective logs of the medications used at weeks $3,6,9$, and 12 for the previous three week period. Mothers were also asked to record whether these were prescribed by a GP or bought over the counter.

COST BURDEN ANALYSIS

In order to calculate the annual total cost to the NHS of infant crying and sleeping problems in
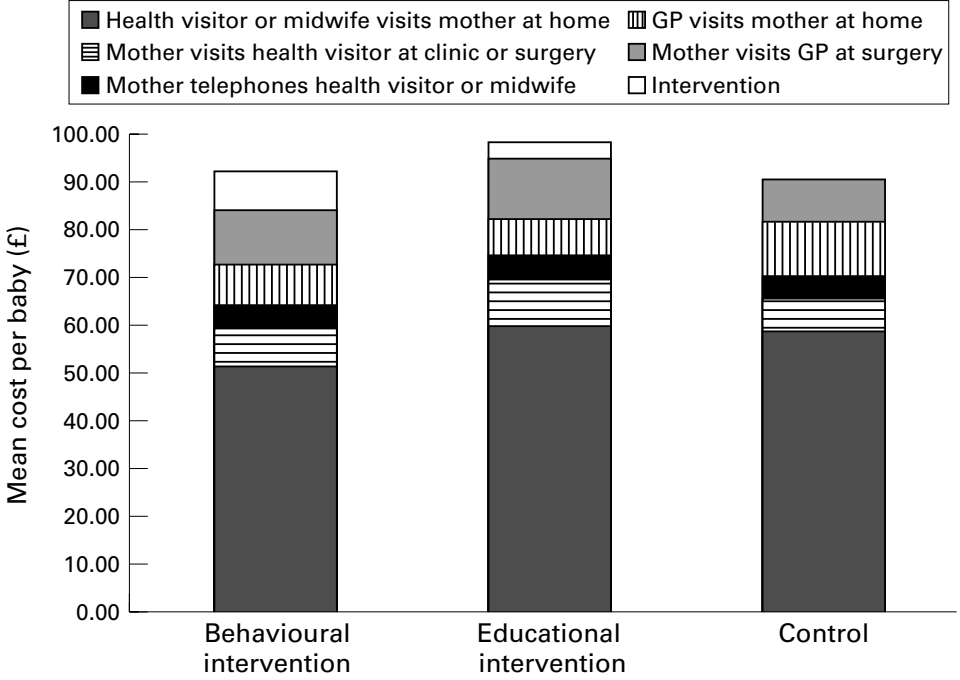

Figure 2 Mean cost per baby for the behavioural intervention, educational intervention, and control groups. the first 12 weeks the mean cost per baby of NHS contacts for infant crying and sleeping problems in the control group was multiplied by the number of live births in the UK in 1997. ${ }^{12}$

\section{COST EFFECTIVENESS ANALYSIS}

Cost effectiveness was measured in terms of the incremental cost per interruption free night gained for behavioural and educational interventions relative to the control. In simple terms this summarises how much extra the intervention costs than the control for one extra uninterrupted night. Mathematically this is calculated as the ratio of incremental costs to incremental effectiveness, or $\left(\mathrm{C}_{\mathrm{i}}-\mathrm{C}_{\mathrm{c}}\right) /\left(\mathrm{E}_{\mathrm{i}}-\mathrm{E}_{\mathrm{c}}\right)$, where $C_{i}$ is the mean cost per baby in each intervention group, $\mathrm{C}_{\mathrm{c}}$ is the mean cost per baby in the control group, $\mathrm{E}_{\mathrm{i}}$ are the mean interruption free nights per baby in each intervention group, and $\mathrm{E}_{\mathrm{c}}$ are the mean interruption free nights per baby in the control group. The incremental cost $\left(\mathrm{C}_{\mathrm{i}}-\mathrm{C}_{\mathrm{c}}\right)$ summarises how much more money each intervention costs over and above the control group. The incremental effectiveness $\left(\mathrm{E}_{\mathrm{i}}-\mathrm{E}_{\mathrm{c}}\right)$ summarises how many more interruption free nights are obtained in each intervention group relative to the control.

Discounting was unnecessary because the time period was less than one year.

SENSITIVITY ANALYSIS

Uncertainty in the cost burden analysis existed surrounding the true number of NHS contacts and hence the mean cost per baby of those contacts. We conducted a sensitivity analysis by calculating the upper and lower 95\% confidence limits around the mean cost per baby of all NHS contacts in the control group. These were multiplied by the total number of live births in 1997 to calculate an upper and lower estimate of the cost burden.

Uncertainty in cost effectiveness analysis existed on two levels: uncertainty in the estimated values of cost effectiveness; and uncertainty concerning the value of the critical cost effectiveness ratio (the maximum cost effectiveness ratio that a decision maker would consider acceptable for implementation or expansion of a particular health care programme). ${ }^{13}$ To consider both levels of uncertainty simultaneously we constructed cost effectiveness acceptability curves using the net 
Table 2 Effectiveness, costs, and cost effectiveness for the behavioural intervention, educational intervention, and control groups

\begin{tabular}{|c|c|c|c|}
\hline & Effectiveness & Costs & Cost effectiveness \\
\hline & Mean (SD) interruption free nights per baby & Mean $(S D)$ costs per baby $(£)$ & \\
\hline Behavioural intervention & $40.22(20.25)$ & $92.45(116.92)$ & \\
\hline Educational intervention & $39.03(22.06)$ & $98.96(127.88)$ & \\
\hline \multirow[t]{2}{*}{ Control } & $37.02(21.83)$ & $90.64(123.30)$ & \\
\hline & $\begin{array}{l}\text { Mean ( } 95 \% \text { CI) interruption free } \\
\text { nights gained }\end{array}$ & $\begin{array}{l}\text { Mean }(95 \% \text { CI) incremental } \\
\text { cost }(£)\end{array}$ & $\begin{array}{l}\text { Incremental cost per interruption free } \\
\text { night gained }(f)\end{array}$ \\
\hline Behavioural intervention versus control & $3.20(-1.73$ to 8.14$)$ & $1.81(-25.17$ to 28.79$)$ & 0.56 \\
\hline Educational intervention versus control & $2.02(-3.07$ to 7.10$)$ & $8.32(-19.93$ to 36.57$)$ & 4.13 \\
\hline
\end{tabular}

benefits approach to describe the relation between the critical cost effectiveness ratio and the probability that the true cost effectiveness ratio fell below the critical cost effectiveness ratio. This was based on the observed mean and standard deviation of differences in costs and effectiveness obtained in the clinical trial. ${ }^{1415}$ Two curves were constructed, one for each intervention relative to the control.

\section{Results}

EFFECTIVENESS

Figure 1 presents the mean interruption free nights per baby per week. Across the 12 week period complete data were returned for $73 \%$ of babies in the behavioural intervention group (150/205), $79 \%$ of babies in the educational intervention group (159/202), and $65 \%$ of babies in the control group (132/203). This loss to follow up was a consequence of the onerous nature of completing the prospective logs for a 12 week period.

The mean interruption free nights per baby across the whole 12 week period were 40.22 for the behavioural intervention group, 39.03 for the educational intervention group, and 37.02 for the control group. Differences between the behavioural and control group were statistically significant at 11 and 12 weeks of age.

costs

Table 1 presents the number and cost of NHS contacts pertaining wholly or in part to infant crying and sleeping problems. The numbers of non-NHS contacts are also presented. These were not included in the economic evaluation because they did not incur a cost to the NHS.

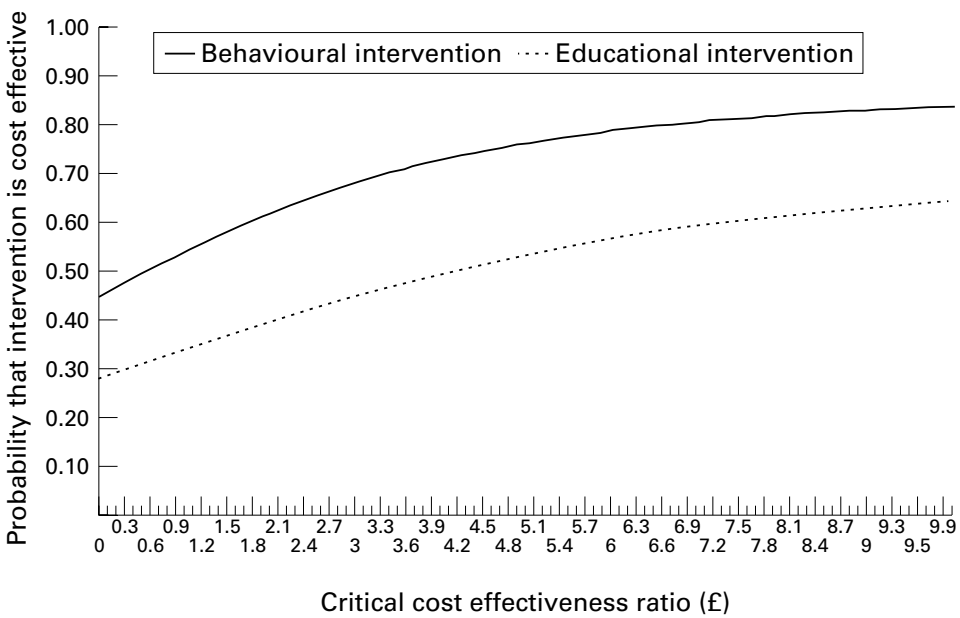

Figure 3 Cost effectiveness acceptability curves: probability that the behavioural and educational interventions are cost effective relative to the control as a function of the critical cost effectiveness ratio.
Medications used for babies prompted by their crying and unsettled behaviour included colic relieving remedies, infant paracetamol, antibiotics, and herbal remedies. These were not included in the economic evaluation because over $99 \%$ were purchased over the counter at zero cost to the NHS. There were no significant differences across study groups.

Combining intervention costs and the cost of NHS contacts, the mean cost per baby was $£ 92.45$ (US $\$ 152.54$ ) for the behavioural intervention group, $£ 98.96$ (US\$163.28) for the educational intervention group, and $£ 90.64$ (US\$149.55) for the control group (fig 2).

COST BURDEN ANALYSIS

The mean cost to the NHS of infant crying and sleeping problems in the first 12 weeks was £90.64 (US\$149.50) per baby. In 1997 there were 725800 live births in the UK. ${ }^{12}$ Therefore the annual total cost to the NHS was £65 786512 (US\$108 547715 ).

The lower $95 \%$ confidence limit around the mean cost per baby was $£ 70.61$ (US $\$ 116.40$ ). On this basis the annual total cost to the NHS was $£ 51248738$ (US\$84 550418 ). The upper $95 \%$ confidence limit was $£ 110.67$ (US $\$ 182.60)$. On this basis the annual total cost to the NHS was £80324286 (US\$132 535 072).

COST EFFECTIVENESS ANALYSIS

Table 2 summarises mean costs and effectiveness per baby for each study group. Incremental costs per interruption free night gained for the behavioural intervention relative to control were $£ 0.56$ (US\$0.92). Incremental costs per interruption free night gained for the educational intervention relative to control were $£ 4.13$ (US $\$ 6.50$ ).

Figure 3 presents the cost effectiveness acceptability curves. These show, for example, that the probability that the behavioural intervention was cost saving relative to the control was 0.45 and that there was a probability of 0.5 that the cost per interruption free night gained of the behavioural intervention relative to control was less than the point estimate of cost effectiveness ( $£ 0.56$ (US $\$ 0.92$ ) per interruption free night gained).

\section{Discussion}

In this study data from a prospective randomised controlled trial were used to estimate the annual total cost to the NHS of infant crying and sleeping problems in the first 12 weeks. We multiplied the mean cost per baby of NHS contacts for infant crying and sleeping problems in the control group of the trial by the 
number of live births and estimated the annual cost burden. With this amount of money it would be possible to employ an additional $2391 \mathrm{E}$ grade nurses for one year, ${ }^{11}$ treat 1284 patients with HIV for life, ${ }^{16}$ or pay for $1360982950 \mathrm{mg}$ doses of sildenafil. ${ }^{17}$

The results were calculated by extrapolating total costs from the relatively small number of total births in the control group to the UK population. This was based on the assumption that the control group was representative of the general population with reference to infant crying and sleeping problems. It was not possible to ascertain the validity of this assumption because this study has produced the largest and most comprehensive dataset of infant crying and sleeping problems in the first 12 weeks in the UK to date. One might speculate that the entry criteria might have led to high risk babies being excluded from the trial. On this basis the cost burden estimates would underestimate the true cost. The results of the cost burden analysis should be treated with caution. However, since no estimates currently exist of the cost of infant crying and sleeping problems to the NHS, these results should be viewed as a useful preliminary estimation of the true cost.

Additionally, the cost burden estimates presented in this study comprised only costs to the NHS. If a societal perspective were adopted, this would warrant inclusion of additional non-NHS direct costs, such as the cost of non-NHS contacts (with friends, relatives, and telephone helplines; see table 1), and the cost of medications bought over the counter. Indirect costs (for example, lost productivity because of work absences) would also be included. One would therefore expect the total cost to society of infant crying and sleeping problems in the first 12 weeks to be significantly greater than the cost burden estimates presented here.

We also assessed the cost effectiveness of behavioural and educational interventions aimed at preventing infant crying and sleeping problems relative to usual services. Incremental costs per interruption free night gained for the behavioural intervention relative to control were $£ 0.56$ (US\$0.92). This means that the extra cost of obtaining an extra interruption free night with the behavioural intervention relative to the control was $56 \mathrm{p}$. Incremental costs per interruption free night gained for educational intervention relative to control were $£ 4.13$ (US\$6.80).

Breaking down the cost effectiveness ratios into their component parts (table 2), the incremental cost of the behavioural intervention relative to the control was $£ 1.81$ (US\$2.99) per live birth and the incremental effectiveness was 3.20 interruption free nights gained. For the educational intervention relative to the control these figures were $£ 8.32$ (US\$13.70) and 2.02, respectively. Differences in effect between the behavioural and control group were statistically significant at 11 and 12 weeks of age. The behavioural intervention therefore incurred a small additional cost and produced a small significant additional benefit. There was no such statistically significant difference in effect between the educational intervention and the control. This highlights one important finding of this study: the apparent lack of effect of the educational intervention. The production and distribution of information booklets relating to management of crying and sleeping problems and provision of contact details for voluntary support groups had little impact on infant crying and sleeping problems in the first 12 weeks relative to standard existing services. Nonetheless, it is up to health care decision makers to judge the attractiveness of these strategies and decide whether the extra costs are worth the extra benefits.

A noticeable feature of our study has been the lack of previous research on the cost of infant crying and sleeping problems and the cost effectiveness of strategies for managing these problems. Although measurement of cost and cost effectiveness pose many problems, it is becoming increasingly difficult to avoid in a world of scarce resources and underfunded health care systems. We have attempted to initiate discussion on the application of economic evaluation to infant crying and sleeping problems, and to provide a framework for such evaluation.

This study was funded by the Department of Health (reference MCH 0906). We would like to thank Rona McClandish, Ali McGuire, Miranda Mugford, and Maria Raikou for their comments.

1 Forsyth BW, Leventhal JM, McCarthy PL. Mothers' perceptions of problems of feeding and crying behaviour. perceptions of problems of feeding

2 St James-Roberts I, Halil T. Infant crying patterns in the first year: normal community and clinical findings. $f$ Child Psychol Psychiatry 1991;32:951-68.

3 St James-Roberts I, Hurry J, Bowyer J. Objective confirmation of crying durations in infants referred for excessive crying. Arch Dis Child 1993;68:82-4.

4 Scott G, Richards $M$. Night waking in infants: effects of providing advice and support for parents. $\mathcal{F}$ Child Psychol Psychiatry 1990;31:551-67.

5 Pinilla T, Birch LL. Help me make it through the night: behavioural entrainment of breast-fed infants' sleep patterns. Pediatrics 1993;91:436-44.

6 Wolke D, Gray P, Meyer R. Excessive infant crying: a controlled study of mothers helping mothers. Pediatrics 1994;94:322-32

7 Sleep J, St James-Roberts I, Morris S, Gillham P, Owen C. A randomised control trial to compare alternative strategies for preventing infant crying and sleep problems in the first 12 weeks (The COSI Study). In submission.

8 Anders T, Keener M. Development courses of night-time sleep-wake patterns in full-term and premature infants during the first year of life. Sleep 1985;8:193-204

9 Minde K, Popiel K, Leos N, Falkner S, Parker K, HandleyDerry $M$. The evaluation and treatment of sleep disturbances in young children. I Child Psychol Psychiatry 1993;34:521-33.

10 St James-Roberts I, Sleep J, Morris S, Owen C, Gillham P. Use of a behavioral program in the first three months to prevent infant crying and sleeping problems. In submission.

11 Netten A, Knight J, Dennett J, Cooley R, Slight A. A ready reckoner for staff costs in the NHS. Canterbury: University of Kent, 1998.

12 Office for National Statistics. Annual abstracts of statistics. London: The Stationery Office, 1998.

13 UK Prospective Diabetes Study Group. Cost-effectiveness analysis of improved blood pressure control in hypertensive patients with type 2 diabetes: UKPDS 40. BMF 1998;317: $720-6$.

14 Van Hout BA, Al MJ, Gordon GS, Rutten FF. Costs, effects and c/e-ratios alongside a clinical trial. Health Econ 1994; 3: 309-19.

15 Briggs A, Fenn P. Confidence intervals or surfaces? Uncertainty on the cost-effectiveness plane. Health Econ 1998;7: 723-40.

16 Hughes D, Morris S. The cost-effectiveness of condoms in the prevention of HIV infection in England and Wales: should condoms be available on prescription? F Health Serv Res Policy 1996;1:205-11.

17 British Medical Association and the Royal Pharmaceutical Society of Great Britain. British National Formulary 37. Society of Great Britain. British National
London: British Medical Association, 1999. 